\author{
Sara Pellegrini \\ International Itard Study Center, Chiaravalle, Italy \\ Lexiplus Start Up, Civitanova Marche, Italy \\ e-mail: nuove2020@gmail.com
}

\title{
Slowness and speed of execution as a functional indicator in music therapy
}

\begin{abstract}
The understanding of the symptoms, phenomena and treatment derived from the alteration of brain functions undoubtedly deserves the widest reflection on the interaction between brain and behavior, the inter-reaction of dysfunctions and therefore the interpretation of brain effects and plasticity in relation to the environment and musical treatment.
\end{abstract}

Keywords: rhythmicity, fluidity, working memory

\section{Introduction. Predictions for future actions}

Science has long known about the neuro-anatomical pathways, cortical areas, neurotransmitters and receptors. However, knowledge today dedicates its greatest interest to understanding of the neural networks, such as those responsible for asymmetric brain control of cognitive functions, considered significant also because scientists believe it is interesting that around 9-11 years old the different cortical areas cover functions very different from each other. The brain that works changes continuously and dynamically to explore.

So functionality is subject to reorganization of all functions to generate praxis, attention, control of time, emotional and social behavior, language and communication, and behavior in general. 
The behavior go always in resonates, even when we observe or listen to the actions of another individual without making any purposeful movement. The observation of the actions favors the imitation, the understanding of the motor behavior of others and the learning of the actions themselves.

The finalized actions or praxies affect all behavior and therefore also the language since both are part of neuro-behavior and they are directly related to knowledge. This means that we can perform feedbacks and actions, since our brain evaluates when to adjust, when to undertake a task and then to anticipate, making predictions that prepare the organism for relatively future actions.

This property is largely entrusted to meaningful memory or experience, aided by the observation of the environment that it captures and detects strings of meaning. It happens in fact that the memory through its functions retains the events to fix them in the experience, understanding their succession according to the situation, as well as the plural symbolic ways of expressing and modifying the facts. Memory is in fact implicated in the awareness of every subject when something happens right at the moment in which we are concentrated or with respect to something that takes on a certain meaning. Memory, or rather process memory, is prevalently present in new tasks that require an attention control.

The problem is just that in subjects with poor fluidity there is partial control because they are characterized by excessive slowness or rapidity, or by an altered temporal capacity of the memory to distribute the attention to the selected space in a suitable time. So the poor regulation implies a working memory and an attention capacity that is partly functional. In this regard Baddeley (Baddeley 1981, 1988) believes that working memory represents a central executive and that through a complex articulated system, this acts as a regulator to distribute attention during the unfolding of actions in space and time.

Whatever the action, it is always true that seeing and then wanting to do something or visualize and therefore really doing something implies the use of an object, a reproduction and a transcription. Every action therefore implies the use of an object or a content and the transcription of a trace that restores a personal configuration of the content, that it is concrete or abstract. To do this the mind articulates mainly three elements, such as: 
1) visuocinesthetic or motor program. The dyspraxic subject has difficulty adjusting its manual movements with respect to the orientation, position and size of the object;

2) visuospatial, implies forms and direction (mirror action, the overturning and rotation, implicated in the production of the mental image);

3) executive aspects: fluency, precision and speed.

When these aspects are dysfunctional the subject becomes active in each performance with slow or speed, expressing a partial cognitive and motor regulation, visible in the interruptions, substitutions, strong tiredness, avoidance of the task and irregularities in the performance of the actions in place of uncertain and hesitant actions, as well as poor awareness of actions.

Therefore the subject activates slowly and slowly closes the action out of time: the cerebral energy necessary for the action to slide forward in space and time, or in succession, is activated with a weak alert and therefore the anticipation, necessary to prepare the organism for the appropriate motor program useful for carrying out an effective and efficient action, is not appropriate. This is especially visible in group dances, when crossing the street, in games or sports games, in peer talk, in verbal messages, in activity changes, in orientation, in singing and in music production and reproduction in general.

Music production, like all self-determined activities, calls into question different properties deriving from functional integrations, such as the rhythmicity that intrinsically involves timing and duration.

Rhythm is a property of behavior, which consists in warning one or more interval structures. This is already present spontaneously in infants, from the second year of age it becomes intentional. Only around the age of five children are able to follow and even reproduce a simple binary time, this ability reaches its maximum expression around 6 years. So at six they are able to reproduce a rhythm, to produce a rhythm on their own and to maintain a rhythm without any visual support.

To do this the subject must be able to acquire the minimum sequence. The rhythmic structures from the cognitive and musical point of view find a certain degree of convergence since they represent a succession of elements that are not equal to each other, representing on the spatial level the perception of the shapes of an ensamble. 
This motor and cognitive rhythm sequencing is not exclusive but reverberating.

This allows the cognitive system to spread the information in a multiple and disordered way in order to connect them virtuously in a manner coherent with the existing ones. In fact every experience of meaningful and complete sequential activation is implicitly connected to the sequencing already possessed by all those activities implicated in praxies. The possibility of adjusting learning, already capitalized, also depends on the integrity of cerebellar timing mechanisms, which is mostly partial in subjects with executive difficulties. The timing of the intervals between events and their duration are not the same thing and there is no clear and distinct separation because the neural circuits overlap. Furthermore, the sense of duration does not imply language since duration is also possessed by subjects in the absence of language, as well as by animals.

The sense of rhythm that the subject experiences, starts right from the perception of the correct sound string that occurs in an interval of time and to do this each of us needs the duration of the working memory that allows the memory to process the entire information in a fluid and accurate way. In fact, a subject can reproduce a rhythm in the right relationships but with latency, resulting however altered because it is too fast or messy, lacking in automation and synchronization.

\section{Criteria and practice. The self-paced organization}

In place of normal intellectual skills, the subject performs errors slowly and frequently, so it can be said that this affects the coordinated and fluent exercise of voluntary and automatic actions. However, automation takes place only when there is a good degree of synchronization, which cancels the sequence to globalize in simultaneous and conscious action patterns. These processes therefore involve higher executive functions, such as the processes of ideation, activation, control and coordination of intentional human action and the functioning of the cognitive system, which can be traced back to the pre-frontal lobes, fundamentally explaining the regulation of human functions.

Scientists express a degree of agreement in arguing that the executive improvement of motor and cognitive behavior occurs through practice. 
The question of practice opens up a wide range of reflections on the implementation criteria. To these refer two essential attributes: specificity and intensity. These take the form of strategic indicators (Crispiani, 2016) that guide the training and serve to include a series of factors linked to the context, which inevitably come into play as an intrinsic technological tool for a cognitive and clinical set.

This means that the training, which takes place in an ecological and dynamic regime, for the greater praxic and motor efficiency, may dialogically take into consideration the following indicators.

- Operativism. Attention to actions that are endowed with reversibility and causality, precisely just because the processes of the mind are both operative, because they are directly connected to the structural action on reality, privileging the manipulative operations of construction and deconstruction of the real, that are figurative or abstract deriving from functional additions to interaction with the environment.

- Protagonism. It is the subject that independently activates its own behavior. All that the subject plans with autonomy is capitalized. Differently, as already pointed out by M. Montessori J. M. Itard (Crispiani, 1998) what is induced is not acquired, but is a mere response to stimuli. The training is implicitly configured according to a dynamic and intuitive strategy aimed at making the subject of his own action the protagonist.

- Mentalism. These are cognitive procedures that are characterized by the globalization of actions with the aim of making the subject able to relate every single gesture to the whole action with an ever greater understanding of logical, symbolic and cognitive states. In this case it is a question of understanding mental states, implying the ability to foresee and anticipate behavior and also to perform the mental rotations. Aspects that in dis-executive subjects are critical.

- Organization and sequencing. It is a matter of inducing the subject to sequential continuity and therefore to the execution fluidity, favoring ever longer motor patterns.

- Metacognition, or reflection on saying and doing. If we favor the execution of the entire movement or of the entire motor sequence then we will think the co-organizing about the processing of 
the intuitions and the predictions of the mind, making strategy thanks to the conjunction of cognition, logic and symbols.

- Interactionism. Put the subject in continuous evolution and in constant interaction with the environment, culture, mind, reality and its adaptation. Human functional systems represent an infinite series of integrations of complex and dynamic functions with the anthropological environment. Their dynamism is essential for the functioning and development of future skills. From the point of view of the example of the functional structure, this is not presented as the work of individual functions or single tasks, but as a set of instruments, inter-retro-actions, regulatory spaces, attunement and awareness.

Far from being static and rigid configurations, the functional systems that are involved in this case with musical language follow evolutionary dynamics in response to exogenous actions, such as requests, competition or innovation, which imply the overcoming of functional rigidity for generating the best adaptive response. Adaptation implies a change in the functional structure, since it tends to affect the conditions of effectiveness and efficiency with which the reference activities are carried out. Therefore it is important to think about the general themes of functional systems, the cognitive models of reference and behavior in the context of the musical inter-retro-action.

\section{Functional systems and musical interactions. Cognitive actions}

To the functional system, on the basis of activities carried out specifically in relation to cognition and intensive with respect to praxies, are attribuitable the following musical operating modes because they are transversal to the cognitive regulation:

- distribution of executive frequencies and energy mobilization of advances for cognitive planning;

- the transfer and management of unforeseen events and errors in functional musical operators and distributed cognitive markers;

- the creation of tools and practices for incremental execution of the functionality already owned; 
- the space-time transmission of information on the moments or sequences of the performances and of the possible cognitive and motor variations;

- the development of mechanisms to reduce the imperfections during performance and the associated problems of discomfort of the action in place of the error.

The possibility of giving a cognitive place to critical issues and resources implies the predisposition of operative consciousness mechanisms that can favor the meeting and exchange between the request to perform a musical performance and the respective functional cognitive and motor response.

Such functional devices can be thought of as the structural components of cognitive systems distributed in space and time, configurable in the task organization activities, in the task management space and also in the interruptions during the task, to whose efficiency and effectiveness is strongly connected the ability to find the emergencies of the functional system.

It should also be emphasized that functional systems play a decisive role precisely in the distribution and transfer of sudden contingencies, linked to the task to which the various subjects are subjected, both through the development of self-evident cognitive tools prepared ad hoc, and of cognitive strategies suitable for management of the unforeseen and of the error, with the aim of overcoming the functional rigidity through the music and therefore to generate a musical behavior adapted to the environment.

The relevance of functional interactions in regulation and in infinite combinatorics through musical actions, taking into account unforeseen events and risks, lies to a great extent in the planning and learning of information and in the energy cost of functional transactions, which can grow or decrease when increases performance, especially in relation to the overall nature of the operations performed. In fact, through the creation of methods of execution and musical production, necessary for the exchange of actions, functional systems can be generative of the modes of regulation of the exchanges possible starting from the operations requested. So all the functions and their integrations contribute to define the musical performance, in relation to the context and in relation to the requested model. 
However, the performances are not immobile, so the transmission of the necessary sequences to the advances for the best functional planning must be encouraged. In fact, the role played by the functional system in determining the means of execution and functional credit, confers it a key position in transmitting to thought the variations in the requests for functional production established by the trainer.

In addition to what has just been said about functionality, through the language of music, useful information is produced for organizations of the various functional activities that underlie the characteristics of praxia that guiding the quality traits such as continuity, self-evidence and adequacy.

Also the feasibility of the task turns out to be useful to the production of the musical language and to convey the musical language itself, going therefore to constitute the essential conditions within the process of assumption of decision of the task as a less uncertainty on the part of the subjects that are in formation. The trainer, through a self-evident motor and cognitive modality, improves the conditions of the proper cognitive and environmental functional regulation, aiming at protecting and promoting the functional efficiency of the human system. It follows that imperfections are reduced at the same time during the anticipatory phase of the action useful for the whole and complex processing of the action itself.

The minimization of environmental precautions and more generally of all those aspects in which an informative and therefore organizational dis-comfort are manifested constitutes an essential condition for the purposes of the best possible functioning of the training process, carried out by the functional system.

Lack, fragmentation or discontinuity of the information available to the subjects in training also in the musical field contribute to favoring the non-constancy and the scarce participation in the performance in the presence of high degrees of uncertainty and non-awareness.

This could involve the practice of inadequate or partially correct actions because they are based on a non-adaptive organizational set. This is not very suitable for overcoming the rigidity, ending up to stimulate the resistance of the stickiness deriving from the functional asymmetries or from present dis-praxies.

The harmfulness of the interruption of the task occurs again before the subject has cognitively concluded the globality of the sequence 
presented to then induced him to the visualization of each note by note. The visualization of the individual elements not only does not guarantee dynamic recomposition, but rather even tends to hinder it. At first the globalization process expresses uneven critical issues compared to a deficit or dysfunctional situation that expresses motor impairment. The overcoming stasis due to motor embarrassment expresses the need for a not brief task to allow the greatest possible degree of exercise.

This choice expresses the greater probability of causing adverse succession structures to the fluid and meaningful flow of the elements in succession in space and time. Precisely the fragility due to the presence of these imperfections in the space-time succession in the multiple cognitive processes introduces and justifies the presence of a robust training of prevention and remodeling of the error that through the musical language is concerned with activating cognition with fluidity and accuracy.

\section{Conclusion. How to move the information}

To summarize, I believe that the functional actions indicated below can be structural to the musical treatment that aims at fluency, that is:

- to favour known spontaneous body rhythms to facilitate the environmental synchronization of suitable and known sound supports;

- to bring up on musical and lexical segmentation to practice sequencing;

- to educate to the sequencing of rhythms or rhythmic structures according to an incremental model;

- to bring to actions organized in advance in a sequential manner according to a correct and predictive use;

- to sustain and induce continuity of action and constancy of execution, as this organization expands in space and time according to necessary and directional successions;

- to exercise through The Barrages (Crispiani, Pellegrini, 2006) to the promotion of intuitive and predictive processes through the globalization of incremental graphic schemes;

- to activate and de-activate the incipit and the stopping, that is they stimulate the cerebral electrical activation and its contextual arrest. 
All the activities outlined are characterized by the specificity and intensity of the treatment through musical language according to a coherent and cognitive procedure according to a formative approach.

\section{References}

Baddeley A. D. (1981), The concept of working memory: A view of its current state and probable future development, "Cognition" Aug-Dec;10 (1-3), p. 17-23. Baddeley A. D. (1988), Cognitive psychology and human memory, "Trends in Neurosciences", Volume 11, Issue 4, 1988, p. 176-181.

Crispiani P. \& Pellegrini S. (2006), Dislessia-Disgrafia. Azione 7. I barrages, Parma: Junior.

Crispiani P. (1998), Itard e la pedagogia clinica, Napoli: Tecnodid.

Crispiani P. (2001), Pedagogia Clinica. La pedagogia sul campo, tra scienza e professione, Parma: Junior.

Crispiani P. (2011), Dislessia come disprassia sequenziale, Parma: Junior, Spaggiari. Crispiani P. (2016), The Crispiani Method. Clinic of dyslexia and Dyspraxia, Parma: Junior.

Jeannerod M. \& Arbid M. A. \& Rizzolati G. (1995), Grasping objects: The cortical mechanism of visuomotor transformation, "Trends in Neurosciences", Volume 18, Issue 7, July 1995, p. 314-320.

Njiokiktjen C. \& Chiarenza G. A. (2008), Le disprassie dello sviluppo e i disturbi motori associati, Amsterdam: Suyi Pubblications.

Pellegrini S. (2006), Disturbi specifici di apprendimento e trattamento educativo [in:] Serio N., Moliterni P. (ed.), Qualità della didattica, qualità dell'integrazione. Dal dire al fare, Vasto: Gulliver, pp. 202-217.

Pellegrini S. (2014), Giochi ed artefatti oggettuali ludici per l'attivazione delle funzioni prassico-motorie fluide e sequenziali [in:] Sidoti E. (ed), Dentro la dislessia, Bergamo: Junior, pp. 221-238.

Pellegrini S. (2016), Technology enhanced learnng for specific learnings disorders [in:] D. Siemieniecka (ed.), New technologies in education and Communication, Toruń: NCU, pp. 255-267.

\section{Internet sources}

Istituto Itard Homepage, http://www.istitutoitard.it/pubblicazioni/ 